Publ. RIMS, Kyoto Univ.

12 (1976), 427-437

\title{
On Some Generalizations of the Banach Contraction Theorem
}

Dedicated to the memory of the late Professor Kinjirô KUNUGI

\author{
By
}

Shouro KASAHARA*

A number of generalizations of the well-known Banach contraction theorem are obtained in various directions. However one of them, stated in [5], shows that the Banach contraction theorem still holds for a class of non-metric spaces. This suggests that the notion of metric may not be essential in the Banach contraction theorem and some of its generalizations.

The main purpose of this paper is to show that the Banach contraction theorem and its generalizations due to Diaz and Margolis [1], Luxemburg [7], [8], Maia [9], and the author [5] can be easily derived from a simple fixed point theorem in spaces of type $L$ of Fréchet, which we shall call separated L-spaces. Similar results in non-separated L-spaces will be also stated Moreover as an application to linear spaces, we shall derive a generalization of a theorem of Dotson [2].

1. Let $\omega$ denote the set of all nonnegative integers. A pair $(X, \rightarrow)$ of a set $X$ and a subset $\rightarrow$ of the set $X^{\omega} \times X$ is called an $L$-space ${ }^{1)}$ if the following two conditions are satisfied:

(1) If $x_{n}=x \in X$ for all $n \in \omega$, then $\left(\left\{x_{n}\right\}_{n \in \omega}, x\right) \in \rightarrow$.

(2) If $(s, x) \in \rightarrow$, then $(\boldsymbol{t}, x) \in \rightarrow$ for every subsequence $\mathbb{t}$ of $\boldsymbol{s}$. In what follows, we shall write $s \rightarrow x$ or $x_{n} \rightarrow x$ instead of $(s, x) \in \rightarrow$, and read $s$ converges to $x$, where $s=\left\{x_{n}\right\}_{n \in \omega}$. If $s=\left\{x_{n}\right\}_{n \in \omega}$ is a sequence

Communicated by S. Matsuura, February 19, 1976.

* Department of Mathematics, Kobe University, Nada, Kobe 657, Japan.

(1) This is called a multivalued convergence space by Novák (e.g. see [10]). 
in a set $X$, and if $f$ is a mapping on $X$, then $f(s)$ denotes the sequence $\left\{f\left(x_{n}\right)\right\}_{n \in \omega}$.

Let $(X, \rightarrow)$ be an L-space. It is said to be separated if each sequence in $X$ converges to at most one point of $X$. A mapping $f$ of $X$ into an L-space $\left(X^{\prime}, \rightarrow^{\prime}\right)$ is said to be continuous if $s \rightarrow x$ implies $f(t)$ $\rightarrow^{\prime} f(x)$ for some subsequence $t$ of $s$. By the product space of $(X, \rightarrow)$ and $\left(X^{\prime}, \rightarrow^{\prime}\right)$, we mean the L-space $\left(X \times X^{\prime}, \rightarrow^{\prime \prime}\right)$, where $\rightarrow^{\prime \prime}$ is defined as follows: $\left(x_{n}, y_{n}\right) \rightarrow^{\prime \prime}(x, y)$ if and only if $x_{n} \rightarrow x$ and $y_{n} \rightarrow^{\prime} y$. Let $d$ be a nonnegative extended real valued function on $X \times X$. The L-space $(X, \rightarrow)$ is said to be $d$-complete if each sequence $\left\{x_{n}\right\}_{n \in \omega}$ in $X$ with $\sum_{n=0}^{\infty} d\left(x_{n+1}, x_{n}\right)<\infty$ converges to at least one point of $X$. We need the following

Lemma $\mathbb{1}$. Let $(x, \rightarrow)$ be an L-space which is d-complete for a nonnegative extended real valued function $d$ on $X \times X$. If $(X, \rightarrow)$ is separated, then $d(x, y)=d(y, x)=0$ implies $x=y$ for every $x, y$ in $X$.

Proof. Assume $d(x, y)=d(y, x)=0$, and define $a_{2 n}=x$ and $a_{2 n+1}=y$ for each $n \in \omega$. Then since $\sum_{n=0}^{\infty} d\left(a_{n+1}, a_{n}\right)=0$, we have $a_{n} \rightarrow a$ for some $a \in X$. Hence the sequence $\left\{a_{n}\right\}_{n \in \omega}$ converges to $a$ and $x$ at the same time, and consequently we have $a=x$. Similarly $a=y$.

For each mapping $f$ of a set $X$ into itself, $f^{0}$ stands for the identity mapping of $X$ into itself.

2. We shall begin with the following simple observation, which generalizes a known result in metric spaces.

Theorem $\mathbb{1}$. Let $(X, \rightarrow)$ be a separated L-space which is d-complete for a nonnegative extended real valued function $d$ on $X \times X$, and $f$ be a continuous mapping of $X$ into itself satisfying the following conditions for some $\alpha, \beta$ with $0 \leqq \alpha<1$ and $0<\beta \leqq \infty$.

$$
\begin{aligned}
& \quad d\left(f^{2}(x), f(x)\right) \leqq \alpha d(f(x), x) \quad \text { for every } x \in X \text { with } \\
& d(f(x), x)<\beta .
\end{aligned}
$$


(2) $d(f(a), a)<\beta$ for some $a \in X$.

Then $f$ has a fixed point, and the sequence $\left\{f^{n}(a)\right\}_{n \in \omega}$ convorges to a fixed point of $f$.

Proof. As can readily be seen by induction, $d\left(f^{n+1}(a), f^{n}(a)\right) \leqq$ $\alpha^{n} d(f(a), a)$ for every $n \in \omega$, and so we have $\sum_{n=0}^{\infty} d\left(f^{n+1}(a), f^{n}(a)\right)<$ $\infty$. Hence the $d$-completeness of the space implies that the sequence $s=$ $\left\{f^{n}(a)\right\}_{n \in \omega}$ converges to some $x \in X$. So, by the continuity of $f$, there is a subsequence $t$ of $s$ such that $f(\boldsymbol{t}) \rightarrow f(x)$. But then since $f(\boldsymbol{t})$ is a subsequence of $s$, we have $f(t) \rightarrow x$. Therefore we obtain $f(x)=x$.

As an immediate consequence of this theorem we have a fixed point theorem of Banach s contraction type in L-spaces:

Theorem 2. Let $(X, \rightarrow)$ be a separated L-space rohich is d-complete for a nonnegative extended real valued function $d$ on $X \times X$, and $f$ be a continuous mapping of $X$ into itself satisfying the follorving conditions for some $\alpha, \beta$ rith $0 \leqq \alpha<1$ and $0<\beta \leqq \infty$.

(1) $\quad d(f(x), f(y)) \leqq \alpha d(x, y)$ for every $x, y \in X$ reith $d(x y)<\beta$.

(2) $\quad d(f(a), a)<\beta$ for some $a \in X$.

Then $f$ has a fixed point, and the sequence $\left\{f^{n}(a)\right\}_{n \in \omega}$ converges lo a fixed point of $f$. If in addition

(L) $d(x, y)<\beta$ for all fixed points $x, y \in X$ of $f$, then $f$ has a unique fixed point in $X$.

Proof. It will suffice to show that $f$ has at most one fixed point under the condition (L). Let $x, y \in X$ be fixed points of $f$. Then, since $d(x, y)<\beta$, we have $d(x, y)=d(f(x), f(y)) \leqq \alpha d(x, y)$, and so $d(x, y)$ $=0$. Since $d(y, x)<\beta$ this implies $d(y, x)=0$. Therefore we have $x=y$ by Lemma 1 .

Remark $\mathbb{1}$. Each theorem mentioned above asserts that under certain hypothesis (H), if $d(f(a), a)<\beta$ for some $a \in X$, then $f$ has a fixed point in $X$, and the sequence $\left\{f^{n}(a)\right\}_{n \in \omega}$ converges to a fixed point of $f$. However we can derive easily from each of them a "theorem of alterna- 
tive" (cf. [1]) of the form: for each $x \in X$, the following alternative holds under $(\mathrm{H})$ : either

$1^{\circ} \quad d\left(f^{n+1}(x), f^{n}(x)\right) \geqq \beta$ for all $n \in \omega$, or

$2^{\circ}$ the sequence $\left\{f^{m+n}(x)\right\}_{n \in \omega}$ converges to a fixed point of $f$ for some $m \in \omega$.

Moreover if the space $(X, \rightarrow)$ is of type $L^{*}$ (see Kuratowski [6]), then $2^{\circ}$ can be replaced by the assertion: the sequence $\left\{f^{n}(x)\right\}_{n \in \omega}$ converges to a fixed point of $f$.

The following example shows that Theorem 2, and hence Theorem 1 , is no longer true if $f$ is not continuous.

Example $\mathbb{1}$. Let $X$ denote the closed interval $[0,1]$, and $\rightarrow$ the set of all $(s, x) \in X^{\omega} \times X$ such that $s$ converges to $x$ under the usual topology on $X$. Furthermore, for each $x, y \in X$, let

$$
d(x, y)= \begin{cases}|x-y|, & \text { if } x \neq 0 \text { and } y \neq 0 \\ 1, & \text { otherwise }\end{cases}
$$

Then, it is easy to verify that $(X, \rightarrow)$ is a separated $d$-complete L-space. Consider the mapping $f$ defined by $f(0)=1 / 2$ and $f(x)=x / 2$ for every nonzero $x$ in $X$. We have $d(f(x), f(y)) \leqq(1 / 2) d(x, y)$ for every $x$, $y \in X$, but $f$ is not continuous and has no fixed point.

3. The author introduced the notion of premetric spaces in [5]. However, in order to clarify the relation with generalized complete metric space of Luxemburg [7], we state here an alternative equivalent definition. A pair $(X, d)$ of a set $X$ and a nonnegative extended real valued function $d$ on $X \times X$ is called a premetric space, and $d$ a premetric on $X$, if the following conditions are satisfied:

(1) $d(x, x)=0$ for every $x \in X$.

(2) $d(x, y) \leqq d(x, z)+d(z, y)$ for every $x, y, z \in X$.

We say that ${ }^{(2)}$ a sequence $\left\{x_{n}\right\}_{n \in \omega}$ in a premetric space $(X, d)$ converges to $x \in X$, and write $x_{n} \stackrel{d}{\rightarrow} x$, if $d\left(x, x_{n}\right) \rightarrow 0$ as $n \rightarrow \infty$; obviously $(X, \stackrel{d}{\rightarrow})$ is

(2) Some modifications are made for the sake of simplicity. 
an L-space. A premetric space $(X, d)$ is said to be separated ${ }^{(2)}$ if $(X, \stackrel{d}{\rightarrow})$ is separated. We say that ${ }^{(2)}$ a subset $M$ of $X$ is complete if each sequence $\left\{x_{n}\right\}_{n \in \omega}$ in $M$ satisfying the following condition (C) converges to at least one point of $M$.

(C) For any $\varepsilon>0$, there exists a $k \in \omega$ such that $d\left(x_{m}, x_{n}\right)<\varepsilon$ for every $m, n \in \omega$ with $m \geqq n \geqq k$.

If $(X, d)$ is complete, then $(X, \stackrel{d}{\rightarrow})$ is $d$-complete, since $x_{n} \in X$ and $\sum_{n=0}^{\infty}$ $d\left(x_{n+1}, x_{n}\right)<\infty$ imply that, for each $\varepsilon>0$, there exists a $k \in \omega$ such that $d\left(x_{m}, x_{n}\right) \leqq \sum_{i=n}^{m-1} d\left(x_{i \vdash}, x_{i}\right)<\varepsilon$ whenever $m>n \geqq k$. Note that a generalized complete metric space $\mathrm{s}$ a complete premetric space. We are now in a position to state the following consequence of Theorem 2 .

Corollary 1. ([5]) Let $f$ be a mapping of a separated premetric space $(X, d)$ into itself. Suppose that the following conditions are satisfied for some $\alpha$ with $0 \leqq \alpha<1$.

(1) $d(f(x), f(y)) \leqq \alpha d(x, y)$ for every $x, y \in X$ with $d(x, y)<\infty$.

(2) There exists an $a \in X$ such that $d(f(a), a)<\infty$ and $M=$ $\left\{x \in X \mid d(x, a) \leqq(1-\alpha)^{-1} d(f(a), a)\right\}$ is complete.

Then $f$ has a unique fixed point in $M$ and the sequence $\left\{f^{n}(a)\right\}_{n \in w}$ converges to the fixed point.

Proof. Let $\rightarrow$ denote the induced structure of $\stackrel{d}{\rightarrow}$ into $M$. Then $(M, \rightarrow)$ is a $d$-complete L-space. Now for each $x \in M$, we have

$$
\begin{aligned}
d(f(x), a) & \leqq d(f(x), f(a))+d(f(a), a) \leqq \alpha d(x, a)+d(f(a), a) \\
& \leqq \frac{1}{1-\alpha} d(f(a), a) .
\end{aligned}
$$

Hence the restriction of $f$ into $M$ is a mapping of $M$ into itself, which is continuous. Since $a$ belongs to $M$, we see from Theorem 2 that is suffices to prove the uniqueness of the fixed point of $f$. To this end let $x, y \in M$ be fixed points of $f$. Then since $d(x, a)<\infty$, we have $d\left(x, f^{n}(a)\right) \leqq \alpha^{n} d(x, a)$ for every $n \in \omega$. Consequently the sequence $\left\{f^{n}(a)\right\}_{n \in \omega}$ converges to $x$. This shows that the sequence also converges to $y$, and so we obtain $x=y$. 
Corollary 2. Let $f$ be a continuous mapping of a complete separated premetric space $(X, d)$ into itself, and $d^{\prime}$ be a nonnegative extended real valued function on $X \times X$. Suppose that the following conditions are satisfied for some $\alpha, \beta$ with $0 \leqq \alpha<1$ and $0<\beta \leqq \infty$.

(1) $d(x, y) \leqq d^{\prime}(x, y)$ for every $x, y \in X$.

(2) $\quad d^{\prime}(f(x), f(y)) \leqq \alpha d^{\prime}(x, y)$ for every $x, y \in X$ with $d^{\prime}(x, y)$ $<\beta$.

Then for each $x \in X$ the following alternative holds: either

$1^{\circ} \quad d^{\prime}\left(f^{n+1}(x), f^{n}(x)\right) \geqq \beta$ for all $n \in \omega$, or

$2^{\circ}$ the sequence $\left\{f^{n}(x)\right\}_{n \in \omega}$ converges to a fixed point of $f$. If in particular $d^{\prime}(f(a), a)<\beta$ for some $a \in X$ and if the condition (L) is satisfied, then $f$ has a unique fixed point.

Proof. Since $(X, \stackrel{d}{\rightarrow})$ is $d$-complete, it is $d^{\prime}$-complete by the condition (1). Hence the conclusion follows immediately from Theorem 2 and Remark 1.

The fixed point theorem of Diaz and Margolis [1], and hence those of Luxemburg [7], [8], are obtained from Corollary 2 by letting $(X, d)$ a generalized complete metric space and $d^{\prime}=d$, though Luxemburg's are direct consequences of Theorem 2. The fixed point theorem due to Maia [9] is also a special case of Corollary 2. In fact, it suffices to take two metrics $d, d^{\prime}$ and $\beta=\infty$.

4. We shall now proceed to establish some similar results for nonseparated L-spaces.

Theorem 3. Let $(X, \rightarrow)$ be an L-space which is d-complete for a continuous nonnegative extended real valued function $d$ on the product space $X \times X$ with the property that $d(x, y)=0$ implies $x=y$. If $f$ is a continuous mapping of $X$ into itself satisfying the conditions (1) and (2) of Theorem 1 for some $\alpha, \beta$ with $0 \leqq \alpha<1$ and $0<\beta \leqq \infty$, then $f$ has a fixed point, and the sequence $\left\{f^{n}(a)\right\}_{n \in \omega}$ converges to a fixed point of $f$. 
Proof. By induction, we have

$$
d\left(f^{n+1}(a), f^{n}(a)\right) \leqq \alpha^{n} d(f(a), a)
$$

for every $n \in \omega$. Hence the same argument employed in the proof of Theorem 1 yields that the sequence $s=\left\{f^{n}(a)\right\}_{n \in \omega}$ converges to some $x \in X$, and that $f(\boldsymbol{t}) \rightarrow f(x)$ for some subsequence $\boldsymbol{t}$ of $\boldsymbol{s}$. Therefore the continuity of $d$ implies that $d\left(f\left(f^{n(m)}(a)\right), f^{n(m)}(a)\right) \rightarrow d(f(x), x)$ for some subsequence $\left\{f^{n(m)}(a)\right\}_{m \in \omega}$ of $\boldsymbol{t}$. But $\left(^{*}\right)$ shows that $d\left(f\left(f^{n(m)}(a)\right), f^{n(m)}(a)\right)$ $\rightarrow 0$. Hence $d(f(x), x)=0$, and thus we have $f(x)=x$.

It follows from this result that Theorem 2 also holds for such an L-space.

As the following example shows, there is a non-separated $\mathrm{L}$-space $(X, \rightarrow)$ which is $d$-complete for a continuous nonnegative real valued function $d$ on the product space $X \times X$ with the property that $d(x, y)=0$ implies $x=y$.

Example 2. Let $X=\{0,1\}$, and let $\rightarrow$ denote the set of all ordered pairs $(\boldsymbol{s}, 0)$ and $(\boldsymbol{t}, 1)$, where $\boldsymbol{s}=\left\{x_{n}\right\}_{n \in \omega}$ is a sequence in $X$ satisfying the following conditions ( 0 ) or (1), and $t=\left\{x_{n}\right\}_{n \in \omega}$ is a sequence in $X$ satisfying (1):

(0) There is an $m \in \omega$ such that $x_{n}=0$ for all $n \geqq m$.

(1) There is an $m \in \omega$ such that $x_{n}=1$ for all $n \geqq m$.

Then $(X, \rightarrow)$ is a non-separated L-space. The constant function $d$ on $X \times X$ with value 1 is obviously continuous and satisfies trivially the condition that $d(x, y)=0$ implies $x=y$. It is clear that $(X, \rightarrow)$ is $d$ complete.

It should be noted however that an L-space $(X, \rightarrow)$ is separated if there is a continuous nonnegative extended real valued function $d$ on $X \times X$ satisfying the condition that $d(x, y)=0$ if and only if $x=y$.

The following example shows that Theorem 3 is no longer true if $f$ is not continuous.

Example 3. Consider the L-space $(X, \rightarrow)$ and the mapping $f$ of 
Example 1. $(X, \rightarrow)$ is $d$-complete for the usual metric $d$ on $X$. it is easy to see that $f$ satisfies the inequality

$$
d\left(f^{2}(x), f(x)\right) \leqq \frac{1}{2} d(f(x), x)
$$

for every $x \in X$, but it has no fixed point.

5. In [4], Hanspeter showed that the fixed point theorem of Tychonoff remains valid in certain convergence linear spaces (limitierten Vektorräumen of Fischer [3]). On the other hand, Dotson [2] showed that the postulate of convexity in the fixed point theorem of Schauder can be relaxed for nonexpansive mappings. In this section, we shall prove, as an application of Theorem 2, a fixed point theorem in convergence linear spaces which generalizes the theorem of Dotson. An intermediate result (Lemma 3 below) in our argument shows that Theorem 2 properly involves the Banach contraction theorem.

The linear spaces we shall consider are defined on the field $K$ of real or complex numbers. The neighborhood filter of $0 \in K$ for the usual topology on $K$ will be denoted by $\mathbb{V}$. A subset $B$ of convergence linear space $(E, \tau)$ is said to be bounded if the filter $\mathbf{V} B$ generated by the filter base $\{V B \mid V \in \mathbb{V}\}$ converges to $0 \in E$ (see Hanspeter [4]). A sequence $s$ in $E$ is said to converge to $x \in E$ if so does the filter $\Phi(s)$ generated by $s$. We say that a subset $X$ of $E$ is sequentially compact if each sequence in $X$ has a subsequence converging to at least one point of $X$. A mapping $f$ of $X$ into $E$ is said to be sequentially continuous 'f for every $x \in X$, each sequence in $X$ converging to $x$ has a subsequence $s$ such that $f(s)$ converges to $f(x)$. A subset $X$ of a linear space is said to be star-shaped provided that there is an $a \in X$ such that $x \in X$ and $0<\lambda<1$ imply $\lambda a+(1-\lambda) x \in X$. The main result of this section is the following

Theorem 4. Let $X$ be a sequentially compact star-shaped subset of a separated convergence linear space $(E, \tau)$, and $f$ a sequentially continuous mapping of $X$ into itself. Suppose that there exists a nonempty subset $B$ of $E$ satisfying the following conditions:

(1) The convex hull of $B$ is bounded. 
(2) $x-y \in \lambda B(\lambda>0)$ implies $f(x)-f(y) \in \lambda B$, for every $x, y \in X$.

(3) For each $x \in X$, there exists a real number $\mu$ such that $f(x)-x \in \mu B$.

Then $f$ has a fixed point.

Note that in this theorem, if $(E, \tau)$ is a Hausdorff locally convex space, then (1) can be replaced by the condition that $B$ is bounded.

In order to prove the theorem, we need some lemmas. We say that a subset $X$ of a convergence linear space is sequentially complete $\mathrm{f}$ each sequence $s$ in $X$ which generates a Cauchy filter $\Phi(s)$ converges to some point of $X$.

Remark 2. If $X$ is a sequentially compact subset of a convergence linear space $(E, \tau)$, then $X$ s sequentially complete. In fact, let $s$ be a sequence in $X$ such that $\Phi(s)$ s a Cauchy filter. Then $s$ has a subsequence $t$ converging to some $x \in X$. It is not hard to see that the filter $\Phi(\boldsymbol{s})$ is finer than the filter $\Psi=\Phi(\boldsymbol{t})+\Phi(\boldsymbol{s})-\Phi(\boldsymbol{s})$. Since $\Phi(\boldsymbol{s})$ is a Cauchy filter and $\Phi(\boldsymbol{t}) \in \tau x$, the filter $\Psi$ belongs to $\tau x$, and hence so does $\Phi(s)$.

Let $X$ be a subset of a convergence linear space $(E, \tau)$, and let $\stackrel{\tau}{\rightarrow}$ denote the set of all $(s, x) \in X^{\omega} \times X$ with $\Phi(s) \in \tau x$. Then $(X, \rightarrow)$ is an L-space. For a nonempty subset $B$ of $E$, define a nonnegative extended real valued function $d_{B}$ on $E \times E$ by

$$
d_{B}(x, y)=\inf \{\lambda>0 \mid x-y \in \lambda B\}
$$

for every $(x, y) \in E \times E$. The restriction of $d_{B}$ into $X \times X$ will be also denoted by $d_{B}$.

Lemma 2. If $X$ is a sequentially complete subset of a convergence linear space $(E, \tau)$, and if $B$ is a nonempty subset of $E$ with bounded convex hull $\Gamma(B)$. then the L-space $(X, \stackrel{\tau}{\rightarrow})$ is $d_{B}$-complete.

Proof. Assume $\sum_{n=0}^{\infty} d_{B}\left(x_{n+1}, x_{n}\right)<\infty$, where $s=\left\{x_{n}\right\}_{n \in \omega} \quad$ s a sequence in $X$, and let $\varepsilon$ be an arbitrary positive real number. Then there 
is a $k \in \omega$ such that $\sum_{i=m}^{n-1} d_{B}\left(x_{i+1}, x_{i}\right)<\varepsilon$ for every $m, n \in \omega$ with $k \leqq m<n$. Let $\varepsilon_{m}, \varepsilon_{m+1}, \cdots, \varepsilon_{n-1}$ be real numbers for which we have $\sum_{i=m}^{n-1} \varepsilon_{i}<\varepsilon$ and $d_{B}\left(x_{i+1}, x\right)<\varepsilon_{i}$ for each $i \in N=\{m, m+1, \cdots, n-1\}$. Then for each $i \in N$, we can find a positive real number $\lambda_{i}<\varepsilon_{i}$ such that $x_{i+1}-x_{i} \in \lambda_{i} B$. Hence we have

$$
\begin{aligned}
x_{n}-x_{m} & \in \lambda_{m} B+\lambda_{m+1} B+\cdots+\lambda_{n-1} B \\
& \subset\left(\lambda_{m}+\lambda_{m-1}+\cdots+\lambda_{n-1}\right) \Gamma(B) \subset V_{\varepsilon} \Gamma(B),
\end{aligned}
$$

where $V_{\varepsilon}$ denotes the set of all $\xi \in K$ with $|\xi| \leq \varepsilon$. This shows that $\left\{x_{n} \mid n \geqq k\right\}-\left\{x_{n} \mid n \geqq k\right\} \in V_{\varepsilon} \Gamma(B)$. Therefore the filter $\Phi(s)-\Phi(s)$ is finer than the filter $\mathbb{V} \Gamma(B) \in \tau 0$, and so $\Phi(s)-\Phi(s) \in \tau 0$. Hence $\Phi(s)$ is a Cauchy filter. Thus $\Phi(s) \in \tau x$ for some $x \in X$.

Lemma 3. Let $X$ be a sequentially complete subset of a separated convergence linear space $(E, \tau)$, and $f$ a sequentially continuous mapping of $X$ into itself. Suppose that there exist a nonempty subset $B$ of $E$ with bounded convex hull and an $\alpha$ with $0 \leqq \alpha<1$ satisfying the following conditions:

(1) $x-y \in \lambda B(\lambda>0)$ implies $f(x)-f(y) \in \alpha \lambda B$, for every $x, y \in X$.

(2) $f(a)-a \in \beta B$ for some $a \in X$ and for some real number $\beta$. Then $f$ has a fixed point, and the sequence $\left\{f^{n}(a)\right\}_{n \in \omega}$ converges to a fixed point of $f$.

Proof. Evidently one can assume $\alpha>0$ and $\beta>0$. Suppose that $\alpha d_{B}(x, y)<d_{B}(f(x), f(y))$ for some $x, y \in X$. Then we can choose an $\varepsilon$ with $\alpha d_{B}(x, y)<\varepsilon<d_{B}(f(x), f(y))$. Hence $x-y \in \lambda B$ for some $\lambda$ with $d_{B}(x, y) \leqq \lambda<\varepsilon / \alpha$. So it follows from (1) that $f(x)-f(y) \in \alpha \lambda B$, which implies $d_{B}(f(x), f(y)) \leqq \alpha \lambda<\varepsilon<d_{B}(f(x), f(y))$, a contradiction. This establishes that $d_{B}(f(x), f(y)) \leqq \alpha d_{B}(x, y)$ for every $x, y \in X$. Obviously $d_{B}(f(a), a)<\infty$. Thus Lemma 2 and Theorem 2 yield the conclusion.

Proof of Theorem 4. Since $X$ is star-shaped, there is an $a \in X$ such that $x \in X$ and $0<\lambda<1$ imply $\lambda a+(1-\lambda) x \in X$. For each nonzero $n \in \omega$, define mapping $f_{n}$ of $X$ into itself by

$$
f_{n}(x)=\frac{1}{n} a+\left(1-\frac{1}{n}\right) f(x)
$$


for every $x \in X$. Clearly each $f_{n}$ is sequentially continuous. If $x, y \in X$, $\lambda>0$ and $x-y \in \lambda B$, then

$$
f_{n}(x)-f_{n}(y)=\left(1-\frac{1}{n}\right)(f(x)-f(y)) \in\left(1-\frac{1}{n}\right) \lambda B .
$$

Since $f(a)-a \in \beta B$ for some real number $\beta$, we have

$$
f_{n}(a)-a=\left(1-\frac{1}{n}\right)(f(a)-a) \in\left(1-\frac{1}{n}\right) \beta B .
$$

Therefore by Remark 2 and Lemma 3, each $f_{n}$ has a fixed point in $X$. Hence the axiom of choice guarantees the existence of a sequence $s$ $=\left\{x_{n}\right\}_{n \in \omega}$ in $X$ such that $x_{0}=a$ and $f_{n}\left(x_{n}\right)=x_{n}$ for every nonzero $n \in \omega$. Since $X$ is sequentially compact, some subsequence $t$ of $s$ converges to a point $x$ of $X$, and so by the sequential continuity of $f$, we can find a subsequence $\left\{x_{n_{i}}\right\}_{i \in \omega}$ of $\ell$ such that the sequence $\left\{f\left(x_{n_{i}}\right)\right\}_{i \in \omega}$ converges to $f(x)$. On the other hand, since

$$
f\left(x_{n_{i}}\right)=\frac{1}{1-\frac{1}{n_{i}}}\left(x_{n_{i}}-\frac{1}{n_{i}} a\right),
$$

the sequence $\left\{f\left(x_{n_{i}}\right)\right\}_{i \in \omega}$ converges to $x$. Thus we have $f(x)=x$.

\section{References}

[1] Diaz, J. B. and Margolis, B., A fixed point theorem of the alternative, for contractions on a generalized complete metric space, Bull. Amer. Math. Soc., 74 (1968), 305-309.

[2] Dotson, Jr., W. G., Fixed point theorems for non-expansive mappings on star-shaped subsets of Banach spaces, J. London Math. Soc. (2), 4 (1971/2), 408-410.

[3] Fischer, H. R., Limesräume, Math. Ann., 137 (1959), 269-303.

[4] Hanspeter, B., Der Satz von Hahn-Banach und Fixpunktsätze in limitierten Vektorräumen, Comm. Math. Helv., 45 (1970), 393-404.

[5] Kasahara, S., A remark on the contraction principle, Proc. Japan Acad., 44 (1968), 21-26.

[6] Kuratowski, C., Topologie I, Mono. Math., XX (1952), Polska Math. Nauk, Warszawa.

[7] Luxemburg, W. A. J., On the convergence of successive approximations in the theory of ordinary differential equations II, Indag. Math., 20 (1958), 540-546.

[8] Luxemburg, W. A. J., On the convergence of successive approximations in the theory of ordinary differential equations III, Nieuw Arch. Wisk. (3), 6 (1958), 93-98.

[9] Maia, M. G., Un'osservazione sulle contrazioni metrich, Rend. Semi. Mat. Univ. Padova, 40 (1968), 139-143.

[10] Novák, J., On some problems concerning multivalued convergences, Czechoslovak Math. J., 14 (89), (1964), 548-561. 
